\title{
Using a Smartwatch with Real-Time Feedback Instructions Improves the Delivery of High-Quality Cardiopulmonary Resuscitation
}

Tsung-Chien Lu, MD, MS ${ }^{1,2}$, Yao-Ting Chang, MS ${ }^{3}$, Te-Wei Ho, PhD ${ }^{4}$, Yi Chen ${ }^{5}$, Yi-Ting Lee ${ }^{5}$, Yu-Siang Wang ${ }^{5}$, Yen-Pin Chen, MD ${ }^{1}$, Chu-Lin

Tsai, MD, ScD ${ }^{1}$, Matthew Huei-Ming Ma, MD, PhD ${ }^{1}$, Cheng-Chung Fang, MD ${ }^{1}$, Feipei Lai, PhD ${ }^{3,4}$, Anne M. Turner, MD, MLIS, MPH ${ }^{2}$

${ }^{1}$ Dept. of Emergency Medicine, National Taiwan University Hospital, Taipei, Taiwan; ${ }^{2}$ Biomedical and Health Informatics, University of Washington, Seattle, USA; ${ }^{3}$ Dept. of Computer Science and Information Engineering, National Taiwan University, Taipei, Taiwan; ${ }^{4}$ Graduate Inst. of Biomedical Electronics and Bioinformatics, National Taiwan University, Taipei, Taiwan; ${ }^{5}$ Dept. of Physics, National Taiwan University, Taipei, Taiwan

\section{INTRODUCTION}

High-quality cardiopulmonary resuscitation (CPR) affects survival after cardiac arrest. We aimed to investigate whether CPR quality can be improved by providing a smartwatch for healthcare professionals with real-time feedback instructions during resuscitation.

\section{METHODS}

An smartwatch app providing real-time audio (Figure 1) and visual feedback (Figure 2) was developed for use in an Android Wear with a built-in speaker [1]. Emergency Department (ED) professionals (of ACLS-certified doctors and nurses) were recruited and randomly allocated to either the interventional group wearing a smartwatch with the preinstalled app, to a control group without the intervention. All participants were asked to perform a two-minute CPR on a Resusci Anne QCPR training manikin using the 30:2 compressionventilation ratio. Beat-to-beat chest compression rate (CCR) and depth (CCD) were recorded using Laerdal PC SkillReporting software (Figure 3).

\section{台大䁹急診醫學部}

Primary outcomes were CCR and CCD (mean $\pm S D$ ) measured on the manikin. Secondary outcome was the percentage (median and IQR) of chest compressions meeting both the guideline-recommended rate (100-120 min-1) and depth (50-60 $\mathrm{mm}$ ) of high-quality CPR during the 2minute period. Differences between groups were evaluated with the t-test or the Mann-Whitney U-test depending on the distribution.

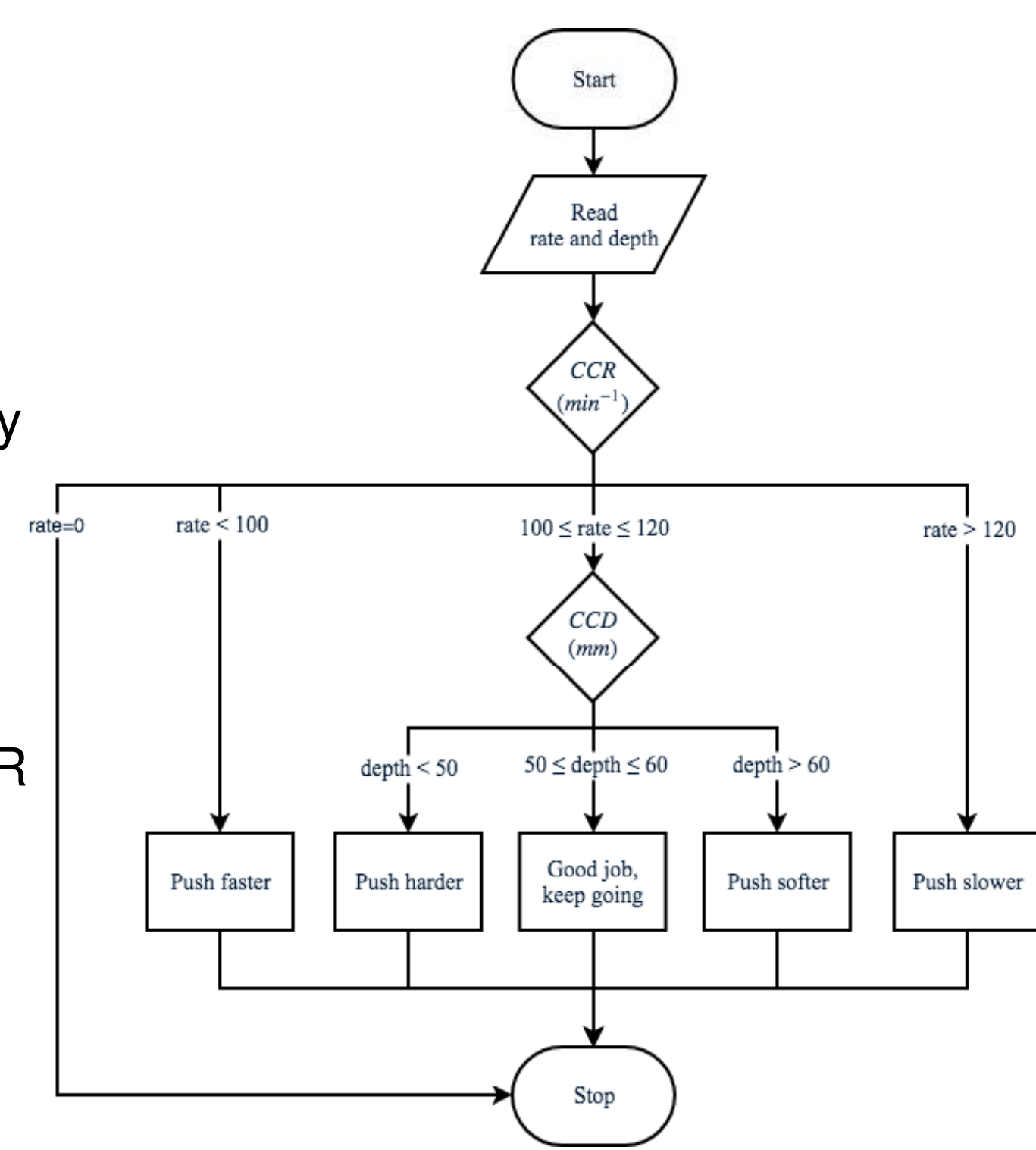

Figure 1. The audio feedback flowchart.

\section{RESULTS}

32 ED professionals were recruited in this preliminary trial. 15 people were assigned to the interventional and 17 to the control group. The compression rates (min-1) were significantly higher (but above the guideline recommendation) in the control group $(123.9 \pm 15.1)$ than the interventional group (109.0 \pm 4.5$)(P<0.05)$. The compression depths $(\mathrm{mm})$ had significant difference between the interventional $(50.0 \pm 7.0)$ and the control groups $(41.6 \pm 7.6)(\mathrm{P}<0.05)$. The percentage (\%) of high-quality CPR was significantly higher in interventional (median 45.1, IQR 7.964.4) than in control group (median 0.0, IQR 0.0-0.2) $(\mathrm{P}<0.05)$.

\section{CONCLUSIONS}

Without real-time feedback, chest compressions tend to be too fast and too shallow. CPR quality may be improved with the assistance of a smartwatch providing real-time feedback instructions in a simulated environment.

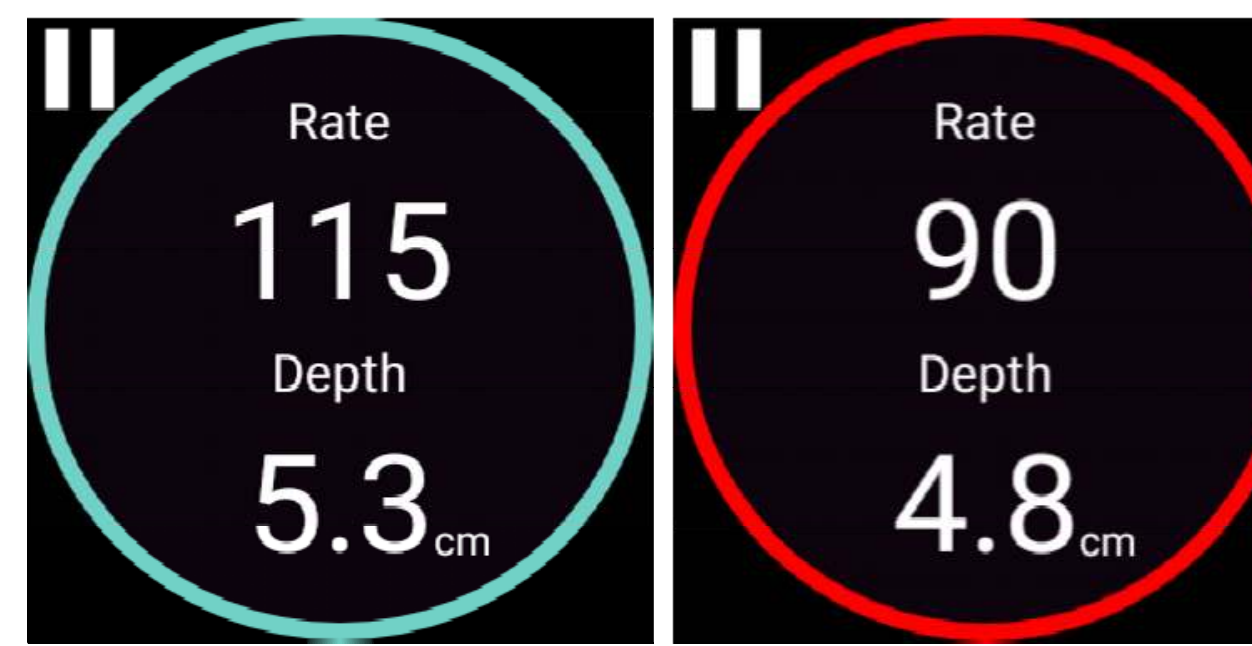

Figure 2. Real time visual feedback displaying CCR and CCD with distinct color shown on the screen of the smartwatch.

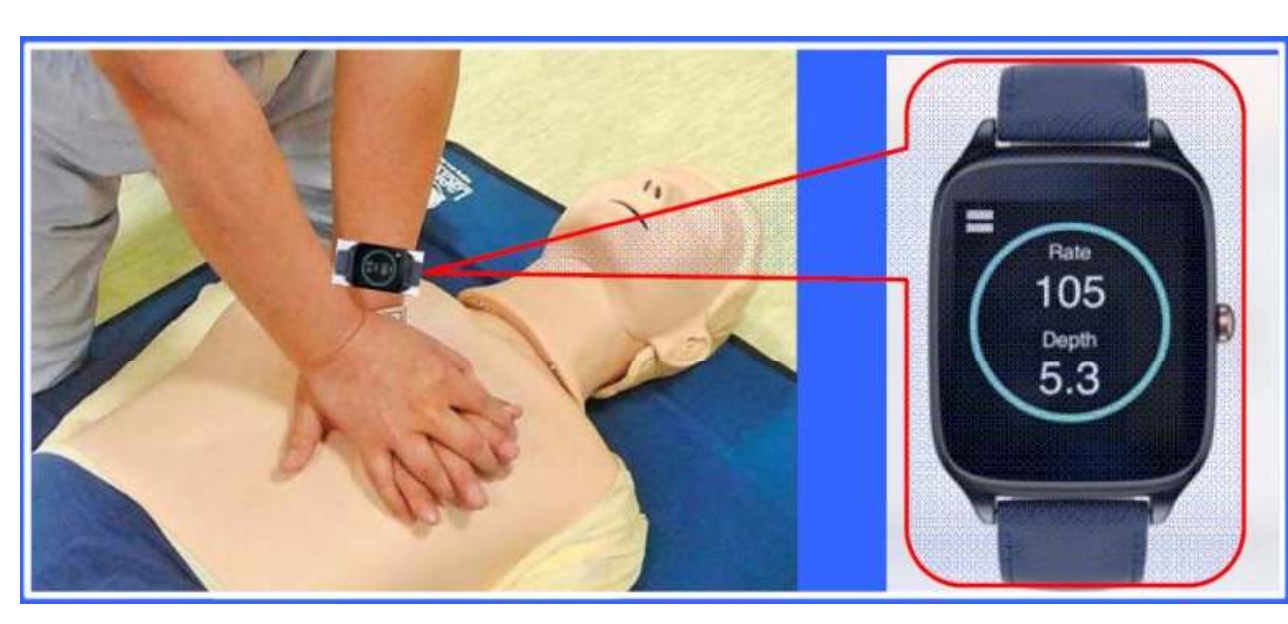

Figure 3. A researcher wearing an Android Wear (ASUS ZenWatch 2) with pre-installed app is performing compression-only CPR on a manikin.

\section{REFERENCET}

1. Lu TC, Chen Y, Ho TW, et al. A Novel Chest Compression Depth Estimation Algorithm Based on a Smartwatch for HighQuality Cardiopulmonary Resuscitation. Poster Session Presented at the AHA Resuscitation Science Symposium (ReSS); 2017 November 11-15; Anaheim, CA, USA.

军 U N I V E R S I T Y O F 